\title{
PMD Tolerance of 10 Gbps Modulated Signals due to SOA-Induced Chirp in SOA Booster Amplifiers
}

\author{
Hodeok Jang, Kyoungsoo Kim, Jaehoon Lee, and Jichai Jeong* \\ Department of Radio Engineering, Korea University, 1, 5Ka, Anam-dong, Sungbuk-ku, Seoul, \\ 136-701, Korea
}

(Received August 20, 2008 : revised October 7, 2008 : accepted October 8, 2008)

\begin{abstract}
We investigated how the polarization-mode dispersion (PMD) tolerance was degraded by semiconductor optical amplifier (SOA)-induced chirp for the $10 \mathrm{~Gb} / \mathrm{s}$ nonreturn-to-zero (NRZ), duobinary NRZ, return-to-zero (RZ), and carrier-suppressed RZ (CS-RZ) modulation formats. The power penalty was calculated as a measure of the system performance due to PMD for a given SOA-induced chirp. Considering only first-order PMD, all modulation formats have a similar PMD tolerance regardless of SOA-induced chirp. On the other hand, when both first- and second-order PMD are considered, the PMD tolerance of all modulation formats with the exception of the CS-RZ modulation format are degraded by SOA-induced chirp. Among all modulation formats considered here, the NRZ modulation format has the PMD tolerance with the highest sensitivity to SOA-induced chirp. When the peak-to-peak chirp induced by SOAs is $0.28 \AA$, its PMD tolerance is degraded up to $4 \mathrm{~dB}$ for a differential group delay (DGD) of 50 ps. However, the PMD tolerance of the CS-RZ modulation format is largely unaffected by SOA-induced chirp.
\end{abstract} Keywords: Polarization mode dispersion, Modulation format, Semiconductor optical amplifier,
Chirp

OCIS codes : (060.0060) Fiber optics and optical communications; (060.2360) Fiber optics links and subsystems; (060.4080) Modulation; (060.4510) Optical communications

\section{INTRODUCTION}

Polarization mode dispersion (PMD) is one of the critical hurdles for next-generation high-bit-rates and long-haul optical transmission systems [1]. Due to the fiber birefringence, a single mode fiber (SMF) supports two orthogonal polarization modes traveling at different velocities along the fiber. The difference in traveling time between the two principal states of polarization (PSP) is called the differential group delay (DGD). Also, PMD has random time-varying characteristics since the fiber birefringence varies along the fiber. DGD is the dominant source of pulse distortion in dispersion managed high bit rate transmission systems. High PMD values are especially interesting since a significant number of installed fibers have PMD values one to two orders of magnitude larger than that of the current state-of-theart.

\footnotetext{
*Corresponding author: jcj@korea.ac.kr
}

The PMD tolerance was investigated for various modulation formats. For first-order PMD only, the duobinary modulation format has the lowest tolerance, since a signal with a larger duty cycle tends to have a larger inter-symbol interference (ISI) [2]. The returnto-zero (RZ) modulation format has the highest tolerance to first-order PMD due to its small duty cycle. On the other hand, RZ-shaped signals are usually less tolerant to second-order PMD than nonreturn-to-zero (NRZ) signals, due to their wide optical spectra. The duobinary modulation format is superior to the NRZ modulation format, in terms of tolerance to first- and second-order PMD due to its narrower power spectrum, whereas the NRZ modulation format has a higher tolerance to firstorder PMD than the duobinary modulation format [3]. These results were obtained under conditions in which the transmission link only involved PMD. When dispersion and nonlinear effects occur in a transmission link with SOAs $[4,5,6]$, it is necessary to investigate PMD tolerance in detail, since SOAs are prospective devices 
for optical fiber transmission systems due to their compact size, ultra wideband gain spectrum, integration with other devices and low cost [4].

In this paper, we investigated first- and second-order PMD tolerance for NRZ, duobinary NRZ, RZ, and CS$\mathrm{RZ}$ modulation formats in $10 \mathrm{~Gb} / \mathrm{s}$ transmission systems with SOAs as booster amplifiers. The modulation formats play an important role in the transmission performance of high speed systems with and without PMD compensation. A modulation format that has a higher tolerance to PMD guarantees improved receiver sensitivity and increased transmission length. Meantime, SOAs as booster amplifiers have the advantage not only of the increase of optical power in the fiber, but also the conversion of positive chirp into negative chirp [7]. In the $1550 \mathrm{~nm}$ wavelength region, self-phase modulation (SPM) induced by high launching power in SMF and negative chirp enables improved-transmission performance for high-bit-rate transmissions in the fiber. We analyzed the PMD tolerance of various modulated signals with SOA-induced chirp, in terms of the receiver sensitivity at $10^{-9}$ bit error rate (BER), which was degraded by a given DGD for optically preamplified receivers. Also, for a specific DGD with the same value as half a bit duration, we investigated the effect of SOA-induced chirp on PMD tolerance.

The paper is organized as follows. In Section II, we present our modeling method for PMD, SOAs, and optical receivers. Section III is dedicated to an analysis of the PMD tolerance of modulation formats with SOAinduced chirp. In detail, we analyze the effect of SOAinduced chirp on the tolerance to first-order PMD only, as well as first- and second-order PMD. For all the simulations in this study, a simulation tool (Photonics CAD) developed by our laboratory was used. Finally, in Section IV we summarize our results and discuss modulation formats that have a higher PMD tolerance to SOA-induced chirp.

\section{MODELS FOR ANALYZING PMD TOLERANCE IN 10 GB/S TRANSMISSION SYSTEMS USING SOAS AS BOOSTER AMPLIFIERS}

\section{A. Modeling of SOAs}

SOAs were modeled using with the time-dependent transfer matrix method (TMM). Since the conventional TMM can't estimate dynamic characteristics of semiconductor optical devices, the time-dependent TMM was employed, to simulate dynamic characteristics of SOAs. Fig. 1 shows a schematic that illustrates wave propagation in a large-signal dynamic SOA model, based on the timedependent TMM. The time-dependent TMM was used to solve the pulse propagation equation in the device [4]. In every section, the complex pulse envelope is cal-

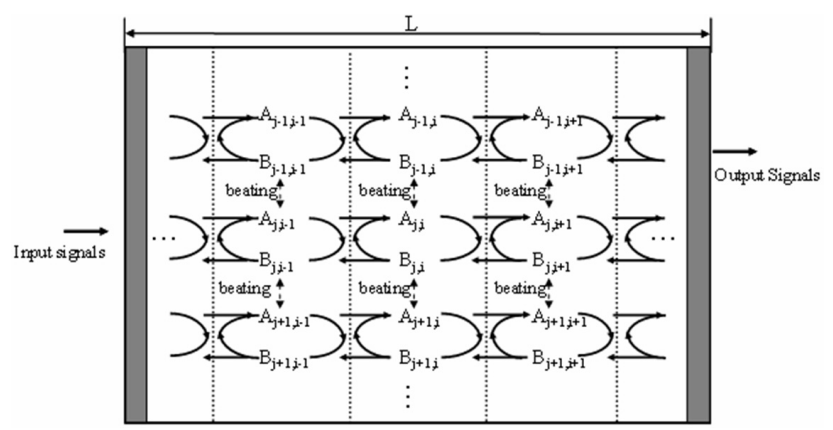

FIG. 1. Schematic diagram that illustrates wave propagation in the large-signal dynamic SOA model based on the time-dependent TMM

culated from consistent solutions to the pulse propagation equations, gain equation, and the rate equation for the carrier density. The amplified spontaneous emission (ASE) noise is calculated from the gain equation and the spontaneous emission factor [8].

\section{B. Modeling of Wave Propagation in Optical Fibers with PMD}

PMD in the fiber can be modeled as a concatenation of several birefringent segments. The input field of birefringent segments is represented by $E_{i n}(t)=E_{i n}(t) j_{i n}$, where $j_{i n}$ is the input Jones polarization vector which depends on the azimuth and ellipticity of the input state of polarization (SOP). The output field is given by [9]

$$
E_{\text {out }}(\omega)=T(\omega) E_{\text {in }}(\omega) j_{\text {in }}=E_{\text {out }}(\omega) j_{\text {out }}(\omega)
$$

where $T(\omega)$ represents the transfer matrix of the fiber and $j_{\text {out }}(\omega)$ is given by the matrix multiplication of the unitary matrix $U(\omega)$ and the input Jones polarization vector $j_{i n}$. $E_{\text {out }}(\omega)$ is obtained by the Fourier transformation of output signals of the lossy and dispersive fiber with nonlinear effects.

In Eq. (1), the unitary matrix $U(\omega)$ can be expressed as: $U(\omega)=R^{-1}(\omega) D(\omega) R(\omega)$, where $R(\omega)$ includes the rotation of PSPs, and the dispersive matrix $D(\omega)$ includes the different propagation speeds in the two PSPs [9].

$$
\begin{aligned}
& R(\omega)=\left[\begin{array}{rr}
\cos k \omega & \sin k \omega \\
-\sin k \omega & \cos k \omega
\end{array}\right] \\
& D(\omega)=\left[\begin{array}{cc}
\exp [j \Delta \tau \omega / 2] & 0 \\
0 & \exp [-j \Delta \tau \omega / 2]
\end{array}\right]
\end{aligned}
$$

where $k$ is a linear PSP rotation with constant angular rate, $\Delta \tau=\Delta \tau_{o}+(\partial \Delta \tau / \partial \omega) \omega$, and $\Delta \tau_{o}$ is GDG. A GDG value of $10 \mathrm{ps}$ was set to be $0.125 \mathrm{ps} / \mathrm{km}$ for a $80 \mathrm{~km}$ long fiber.

The PMD dispersion vector represented by the second- 
TABLE 1. Bandwidth of electrical and optical filters of receivers optimized for various modulation formats

\begin{tabular}{c|c|c}
\hline \hline $\begin{array}{c}\text { Modulation } \\
\text { formats }\end{array}$ & $\begin{array}{c}\text { Electrical } \\
\text { bandwidth }(\mathrm{GHz})\end{array}$ & $\begin{array}{c}\text { Optical } \\
\text { bandwidth }(\mathrm{GHz})\end{array}$ \\
\hline NRZ & 6 & 50 \\
\hline RZ & 6 & 50 \\
\hline Duobinary & 7 & 27 \\
\hline CS-RZ & 6 & 50 \\
\hline
\end{tabular}

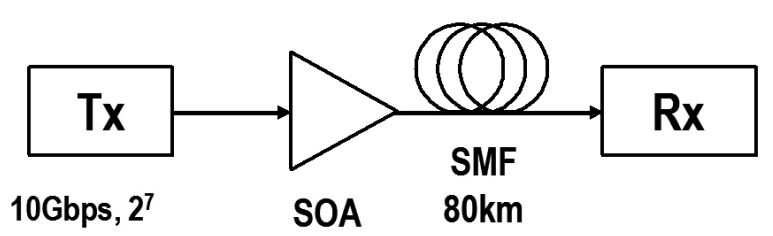

Optically Preamplified Receiver

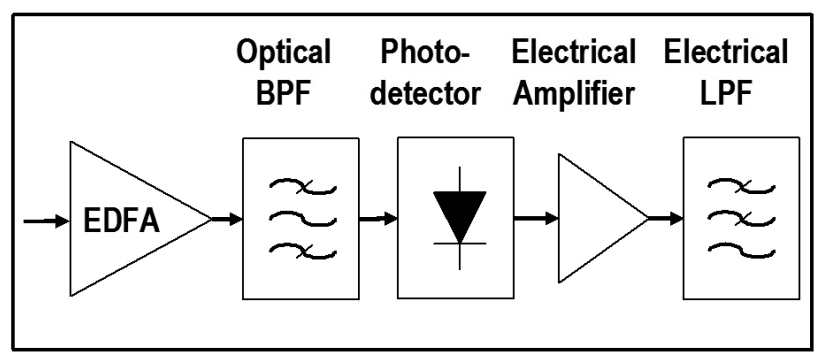

FIG. 2. Schematic diagram of a $10 \mathrm{~Gb} / \mathrm{s}$ transmission system with various modulation formats

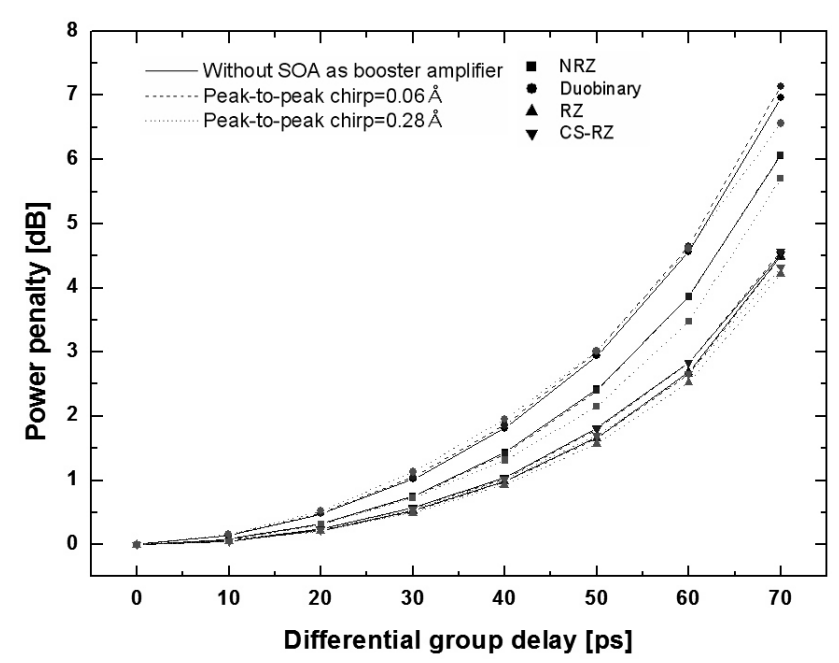

FIG. 3. First-order PMD tolerance of various modulation formats according to the peak-to-peak SOA-induced chirp

order approximation is given by $\vec{\Omega}(\omega)=\left(\Delta \tau_{o}+\omega \cdot \Delta \tau_{\omega}\right)$. $\vec{q}+\Delta \tau_{o} \cdot \omega \cdot 2 \vec{k}$, where $\Delta \tau_{\omega}=\partial \Delta \tau / \partial \omega$ and $2 \vec{k}=\frac{1}{2} \partial \vec{q} / \partial \omega[10]$. Second-order PMD is represented by the two frequency dependent terms: the linear DGD frequency dependence $\left(\Delta \tau_{\omega}\right)$ and the PSP rotation with a constant angular rate $(2|2 \vec{k}|)$. Here, $2|2 \vec{k}|$ perpendicular $\vec{q}$ to causes depolarization [10]. This depolarization is a result of the rotation of PSPs with frequency. The linear DGD frequency dependence induces polarization dependent chromatic dispersion (PCD). PCD eliminates chromatic dispersion under the conditions of first-order PMD compensation and small PSP rotation. In the second-order PMD approximation, the time domain output field obtained by inverting Eq. (1) can be represented as [9]:

$$
\begin{aligned}
\vec{E}_{\text {out }}= & \frac{1}{2 \sqrt{2}}\left\{\left(a \vec{u}^{*}+b \vec{u}\right)\left(E_{\text {out }}^{+}\left(t+\Delta \tau_{o} / 2\right)+E_{\text {out }}^{-}\left(t-\Delta \tau_{o} / 2\right)\right)\right. \\
& +a \vec{u}\left(E_{\text {out }}^{+}\left(t-2 k+\Delta \tau_{o} / 2\right)-E_{\text {out }}^{-}\left(t-2 k-\Delta \tau_{o} / 2\right)\right) \\
& \left.+b \vec{u}^{*}\left(E_{\text {out }}^{+}\left(t+2 k+\Delta \tau_{o} / 2\right)-E_{\text {out }}^{-}\left(t+2 k-\Delta \tau_{o} / 2\right)\right)\right\}
\end{aligned}
$$

where $E_{\text {out }}^{ \pm}(t)=\frac{1}{2 \pi} \int_{-\infty}^{\infty} E_{\text {in }}(\omega) e^{-\alpha z} e^{j\left\{-\bar{\beta}(\omega) z \pm \Delta \tau_{\omega} \omega^{2} / 2\right\}} e^{j \omega t} d \omega$ is the time domain output field, including the loss, chromatic dispersion, and polarization-dependent chromatic dispersion in the fiber, $a=e^{j \theta} \cos (\varepsilon+\pi / 4)$ and $b=e^{j \theta} \cos$ $(\varepsilon-\pi / 4)$, where $\theta$ is the azimuth, and $\varepsilon$ is the ellipticity of the input SOP, $\vec{u}=\left[\begin{array}{ll}1 & j\end{array}\right]$ and $\vec{u}^{*}=\left[\begin{array}{ll}1-j & -j\end{array}\right]$.

\section{Modeling of Receiver}

The receiver model consists of an optical amplifier, followed by a Gaussian band-pass optical filter, a square law detector, and a fifth-order Bessel-Thomson electrical filter [12]. The bandwidth of electrical and optical filters of receivers was optimized for various modulation formats, as shown in Table 1. The shot noise, beat noises generated by signals and amplified spontaneous emission (ASE) noise, and the receiver circuit noise were included in the calculation of the BER, which was optimized with both the threshold level and sampling time [13]. The receiver sensitivity at $10^{-9} \mathrm{BER}$ was used to measure the PMD-induced penalty.

\section{SIMULATION RESULTS AND DISCUSSIONS}

\section{A. First-Order PMD Tolerance due to SOA-Induced Chirp}

Fig. 2 shows a schematic diagram of a transmission system with a $80 \mathrm{~km}$ long fiber. Transmitters for various modulation formats (i.e. NRZ, RZ, duobinary NRZ, and CS-RZ) generate $10 \mathrm{~Gb} / \mathrm{s}$ PRBS signals with $2^{7}$ bits. An SOA was used to amplify the input signals launched into the optical fiber. In the fiber, the dispersion, loss, and fiber nonlinearities were neglected, to consider only PMD. To analyze the first-order PMD tolerance of NRZ, RZ, duobinary NRZ, and CS-RZ modulation formats, we neglected $\Delta \tau_{\omega}$ and $2|2 \vec{k}|$ representing the secondorder PMD. Fig. 3 shows the first-order PMD-induced 

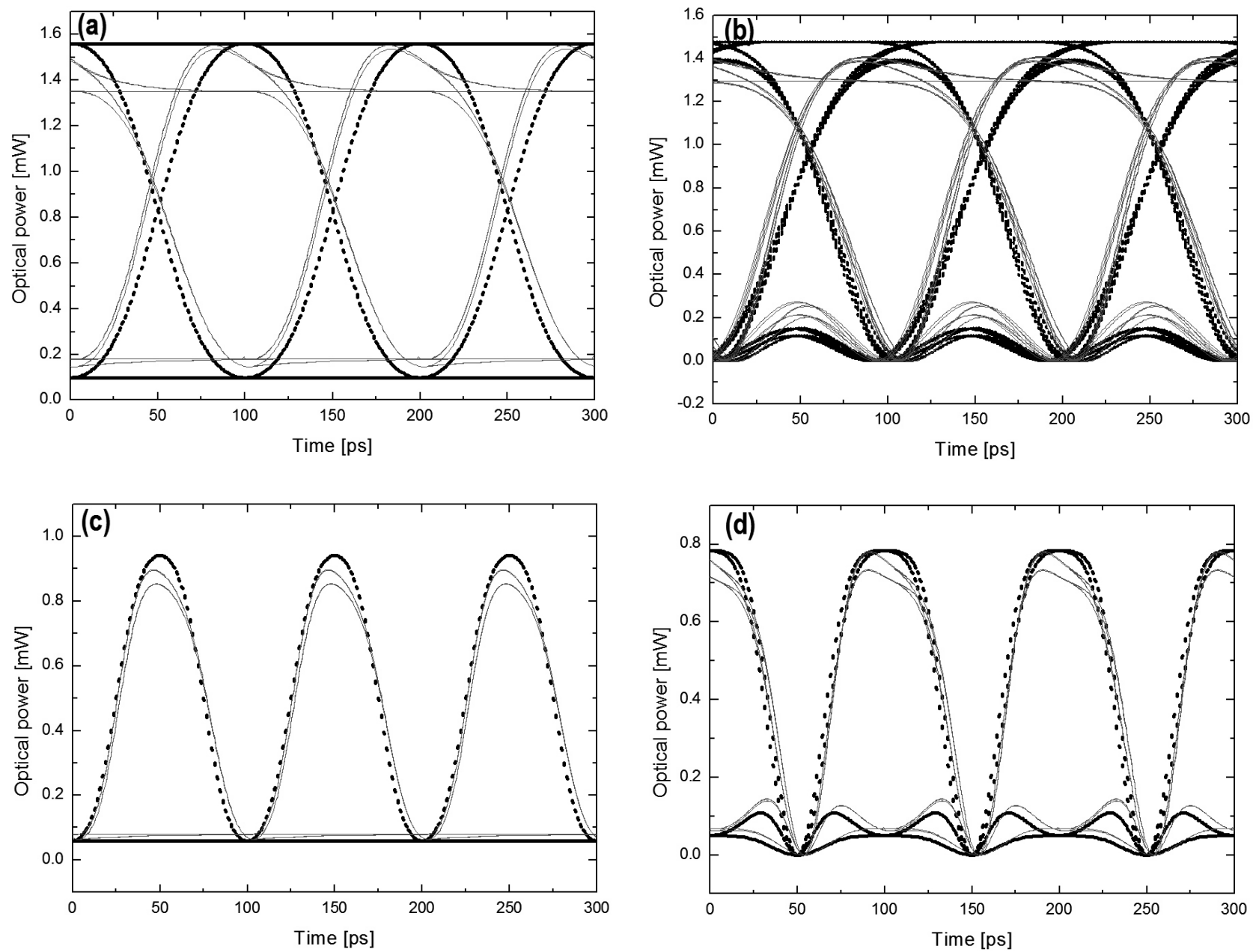

FIG. 4. Optical eye diagrams for various modulation formats of the input (dotted line) and output (red solid line) of SOA with a peak-to-peak chirp of $0.28 \AA$ : (a) NRZ, (b) Duobinary NRZ, (c) RZ, and (d) CS-RZ

TABLE 2. $r m s$ values of $\Delta \tau_{\omega}$ and $2|2 k|$ for a given DGD

\begin{tabular}{c|c|c}
\hline \hline$\Lambda \tau(\mathrm{ps})$ & $\Delta \tau_{\omega}(\mathrm{ps} / \mathrm{GHz})$ & $4 \mathrm{~K}($ degree $/ \mathrm{GHz})$ \\
\hline 10 & 0.02267 & 0.4147 \\
\hline 20 & 0.09068 & 0.8294 \\
\hline 30 & 0.20403 & 1.2441 \\
\hline 40 & 0.36272 & 1.6588 \\
\hline 50 & 0.56675 & 2.0735 \\
\hline 60 & 0.81612 & 2.4882 \\
\hline 70 & 1.11083 & 2.9029 \\
\hline
\end{tabular}

power penalty at $10^{-9} \mathrm{BER}$, for a given SOA-induced chirp with an incident angle of $45^{\circ}$. The incident angle is the angle between the SOP of input signals and PSPs of the fiber. When the incident angle is $45^{\circ}$, the deformation of signals by DGD reaches the maximum, since the inter-symbol interference (ISI) between PSPs of the fiber is maximized. As shown in Fig. 3, all modulation formats have a very similar PMD-induced power penalty, regardless of the peak-to-peak chirp induced by SOA. The first-order PMD tolerance is decided by the duty cycle of signals [2]. A signal with a larger duty cycle has a lower tolerance to the first-order PMD, since it tends to have a larger ISI. However, the duty cycle of signals is unaffected changed by SOA- induced chirp, as shown in Fig. 4. Therefore, the first- order PMD tolerance is largely unaffected by SOA- induced chirp.

\section{B. First- and Second-Order PMD Tolerance due to SOA-Induced Chirp}

To analyze the tolerance of the first- and second-order PMD for various modulation formats, we set $\Delta \tau_{\sigma}$ and $2|2 \vec{k}|$ representing the second-order PMD to their $\mathrm{rms}$ values for a given DGD. The rms values of $\Delta \tau_{\omega}$ $\left(=0.2267 \Delta \tau_{0}^{2}\right)$ and $2|2 \vec{k}|\left(=\sqrt{\frac{\pi}{6}} \Delta \tau_{o}\right)$ can be decided by a theoretical scaling rule based on measurements from several fibers with different mean DGDs and a statistically significant volume of data for each fiber [11]. Table 2 shows the rms value of $\Delta \tau_{\omega}$ and $2|2 \vec{k}|$ for a given DGD. 


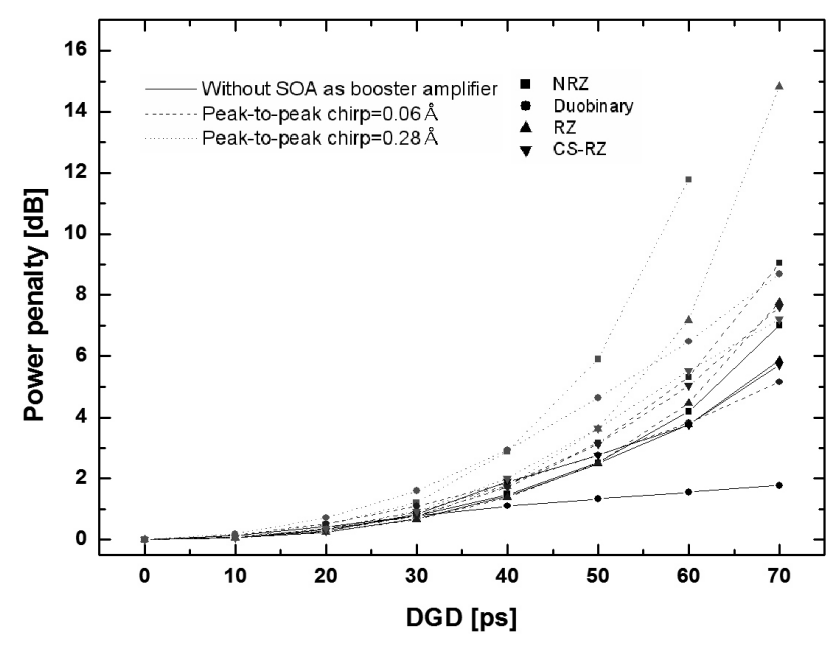

FIG. 5. First- and second-order PMD tolerance of various modulation formats according to the peak-to-peak SOAinduced chirp
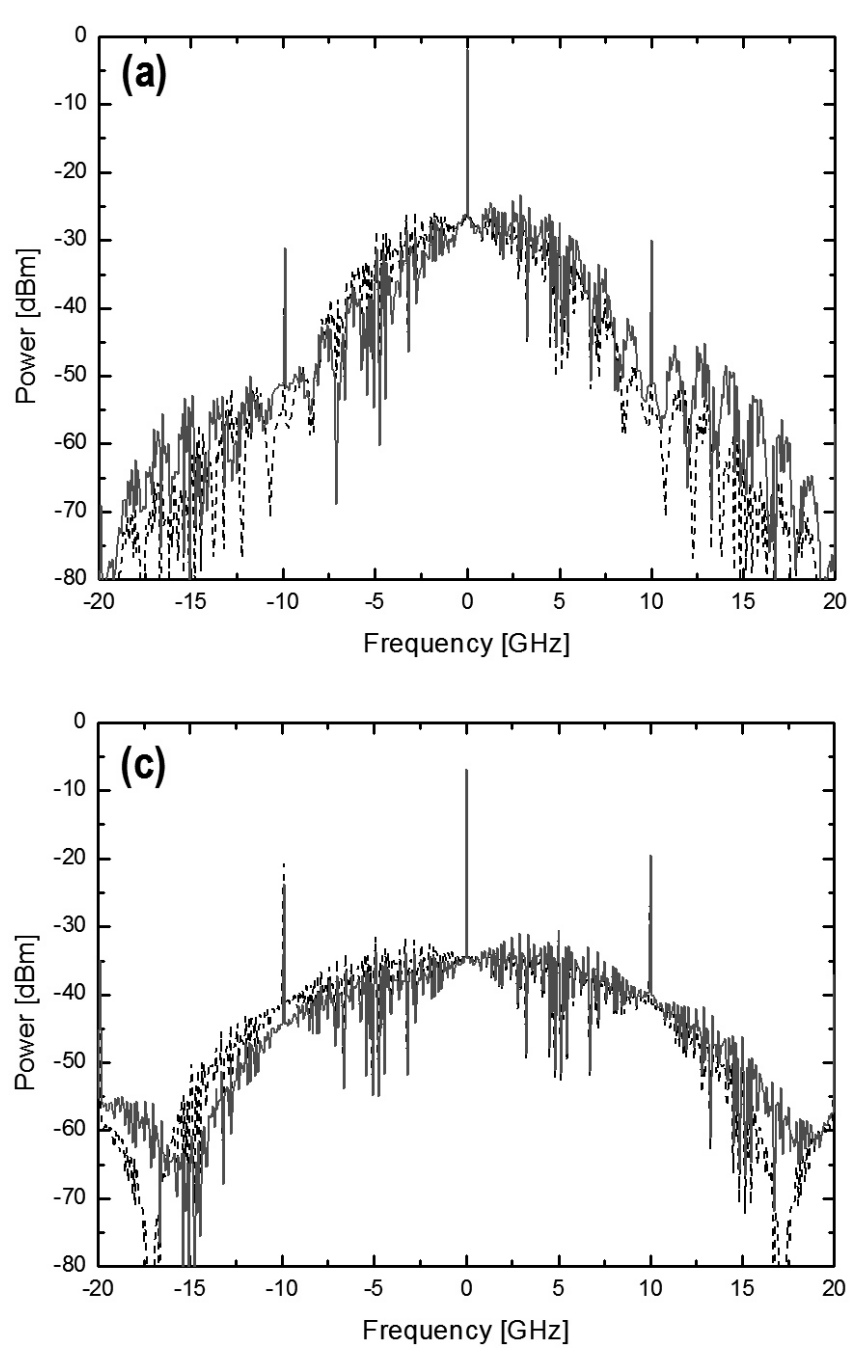

Fig. 5 shows the power penalty due to first- and second-order PMD at $10^{-9} \mathrm{BER}$, for a given SOA-induced chirp. The PMD-induced power penalty of all modulation formats increases as the peak-to-peak SOA-induced chirp increases from $0 \AA$ to $0.28 \AA$. Among all modulation formats considered here, the CS-RZ modulation format has the highest PMD tolerance to SOA-induced chirp, since the spectral components aren't greatly affected by SOA-induced chirp, compared with other modulation formats, as shown in Fig. 6. The figure shows the spectra of all modulation formats for the input and output of the SOA with the peak-to-peak chirp of $0.28 \AA$. The spectral shape of NRZ and duobinary modulation formats varies significantly, due to the spectral regrowth by SOA-induced chirp at a high frequency, but the spectral shape of the CS-RZ modulation format is unaffected by SOA-induced chirp. The second-order PMD tolerance is decided by the spectral width of signals [3]. Therefore, the CS-RZ modulation format has the highest PMD tolerance to SOA-induced chirp, since its spectrum
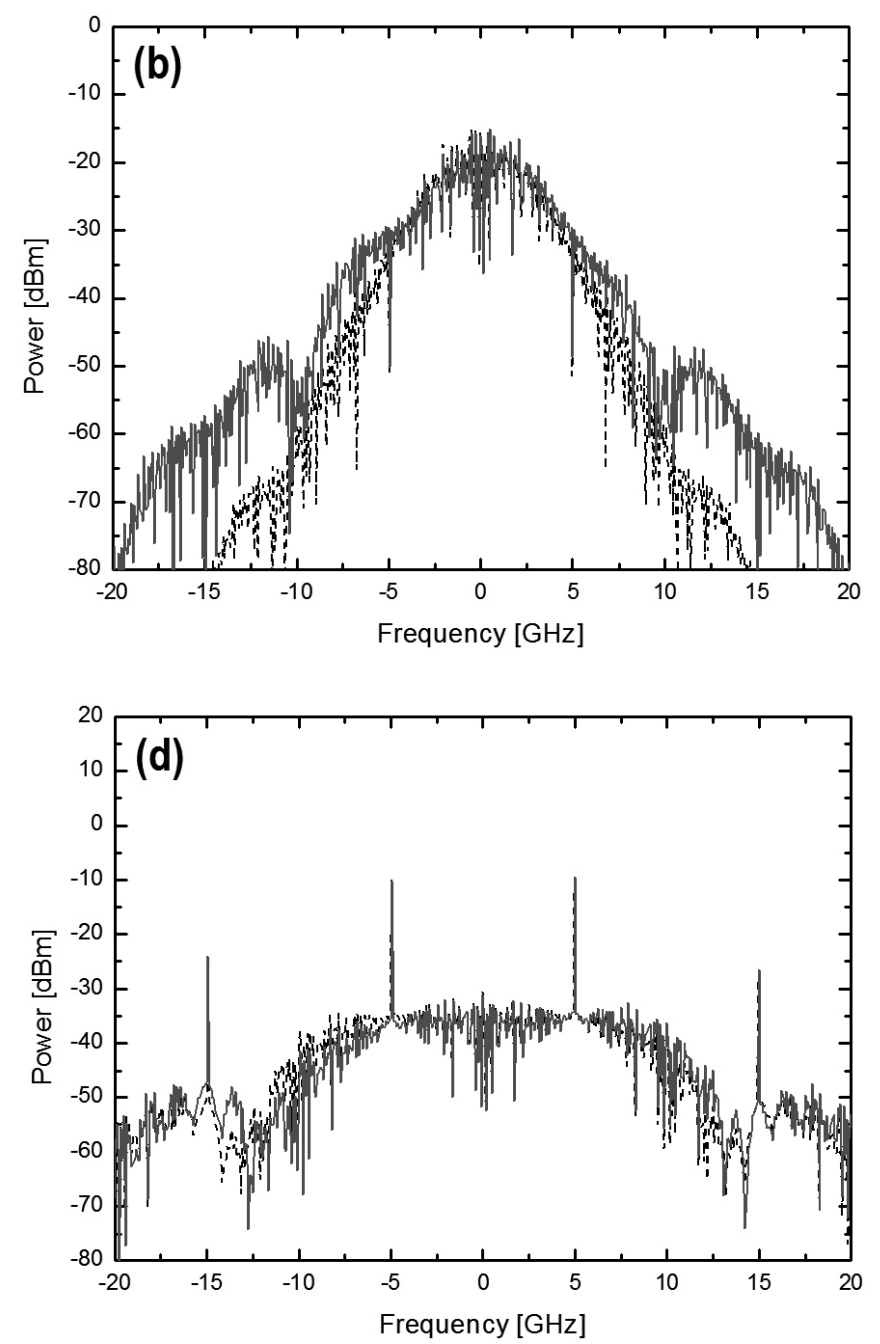

FIG. 6. Spectra for different modulation formats of the input (dotted line) and output (red solid line) of SOA with peak-to-peak chirp of $0.28 \AA$ : (a) NRZ, (b) Duobinary NRZ, (c) RZ, and (d) CS-RZ 


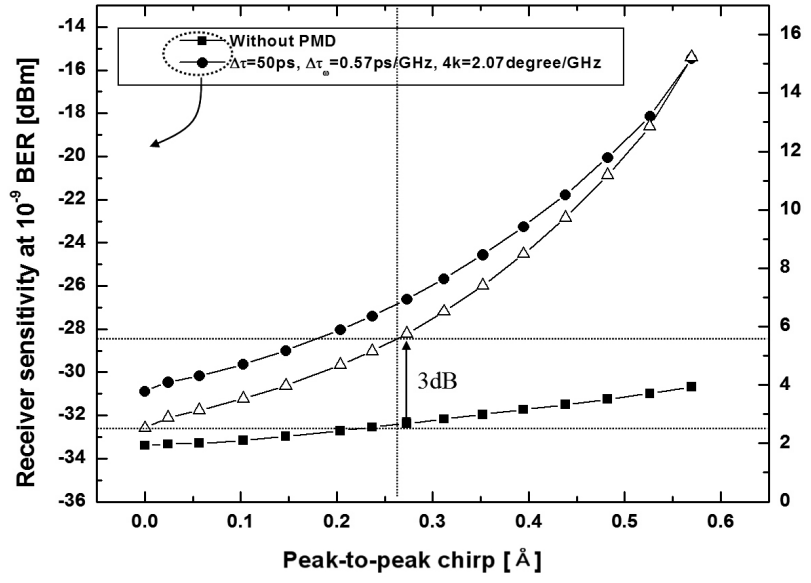

(a)

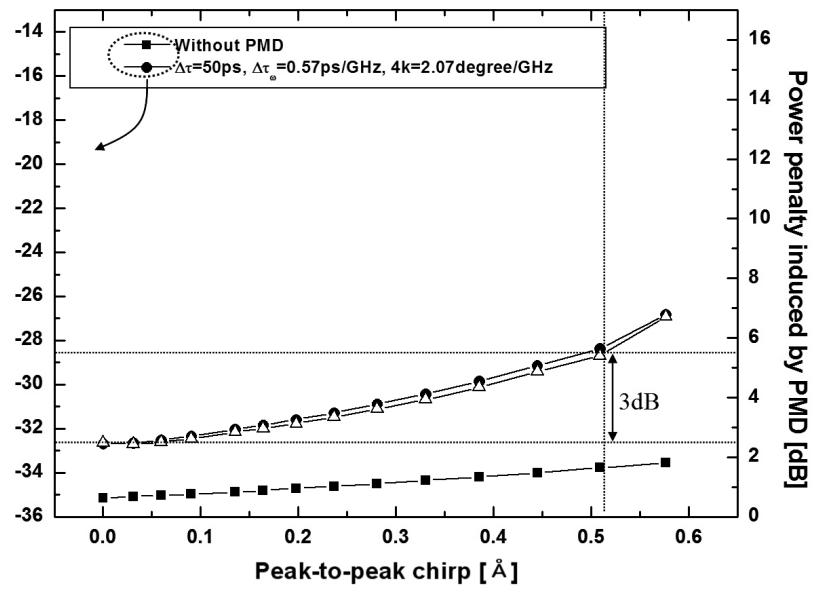

(c)

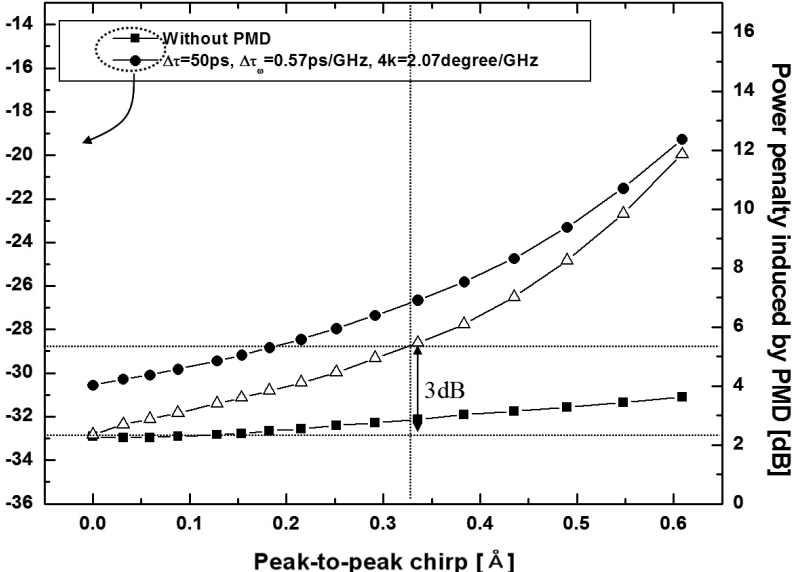

(b)

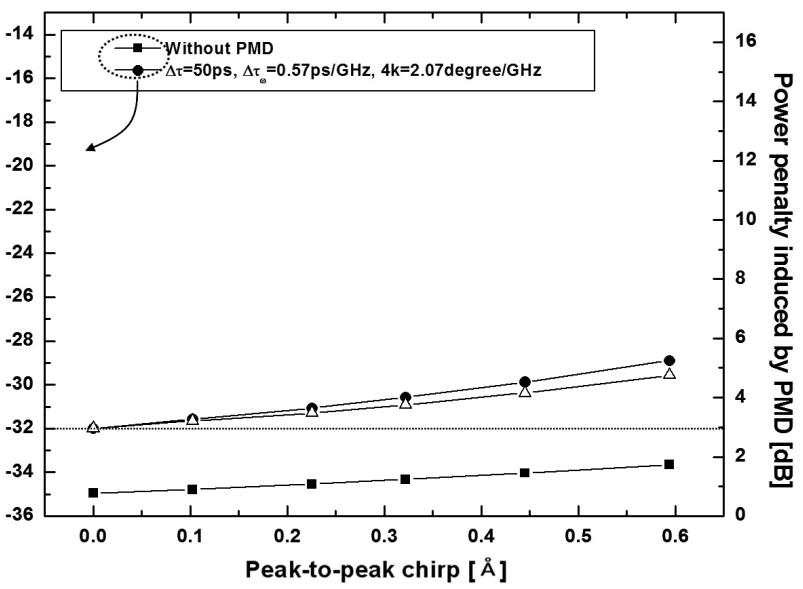

(d)

FIG. 7. Receiver sensitivities at $10^{-9} \mathrm{BER}$ and power penalties induced by first- and second-order PMD as a function of the peak-to-peak SOA-induced chirp; where, DGD $=50 \mathrm{ps}, \Delta \tau_{\varpi}=0.57 \mathrm{ps} / \mathrm{GHz}$, and $2|2 \vec{k}|=2.07^{\circ} / \mathrm{GHz}$; (a) NRZ, (b) Duobinary NRZ, (c) RZ, and (d) CS-RZ

isn't greatly affected by SOA-induced chirp. Where $\mathrm{DGD}=50 \mathrm{ps}, \Delta \tau_{\omega}=0.57 \mathrm{ps} / \mathrm{GHz}$, and $2|\vec{k}|=2.07$ $\circ / \mathrm{GHz}, \mathrm{NRZ}$ and duobinary NRZ signals with the peak-to-peak chirp of $0.28 \AA$ have a PMD-induced power penalty that is $3.4 \mathrm{~dB}$ and $3.3 \mathrm{~dB}$ larger than those without SOA as a booster amplifier, respectively. The RZ modulation format has a PMD-induced power penalty that is $1.1 \mathrm{~dB}$ larger than that without SOA. However, the CS-RZ modulation format has a PMDinduced power penalty that is $0.8 \mathrm{~dB}$ larger than that without SOA.

Fig. 7 shows the receiver sensitivities at $10^{-9}$ BER and the power penalties induced by PMD, according to the peak-to-peak SOA-induced chirp. We calculated the power penalty under constant PMD conditions; Where DGD $=50 \mathrm{ps}$; which is the same value as half a bit duration, $\Delta \tau_{\omega}=0.57 \mathrm{ps} / \mathrm{GHz}$, and $2|2 \vec{k}|=2.07$ / GHz. The NRZ modulation format has a PMD tolerance that is degraded from $2.5 \mathrm{~dB}$ to $17 \mathrm{~dB}$ as the peak-to-peak SOA-induced chirp increases from $0 \AA$ to
$0.6 \AA$. The PMD tolerance is degraded by $3 \mathrm{~dB}$ when the signal has a peak-to-peak chirp of about $0.26 \AA$. The duobinary NRZ modulation format has a PMD tolerance that is degraded from $2.3 \mathrm{~dB}$ to $11.8 \mathrm{~dB}$, as the peak-topeak SOA-induced chirp increases from $0 \AA$ to $0.6 \AA$. The PMD tolerance is degraded by $3 \mathrm{~dB}$ when the signal has a peak-to-peak chirp of about $0.32 \AA$. The RZ modulation format has a PMD tolerance that is gradually degraded from $2.5 \mathrm{~dB}$ to $7 \mathrm{~dB}$ as the peak-to-peak SOAinduced chirp increases from $0 \AA$ to $0.6 \AA$. The peakto-peak chirp, which results in the PMD tolerance being degraded by $3 \mathrm{~dB}$, increases to $0.52 \AA$. However, the CS-RZ modulation format has a PMD-induced penalty that increased by $1.9 \mathrm{~dB}$ only, for a peak-to-peak chirp up to $0.6 \AA$. Consequently, the chirp induced by SOA affects the PMD tolerance of all modulation formats. Specifically, the value of peak-to-peak chirp generating a additional $3 \mathrm{~dB}$ power penalty increases in the following order: NRZ, duobinary NRZ, RZ, and CS-RZ modulation formats. Therefore, in terms of the additional $3 \mathrm{~dB}$ power 

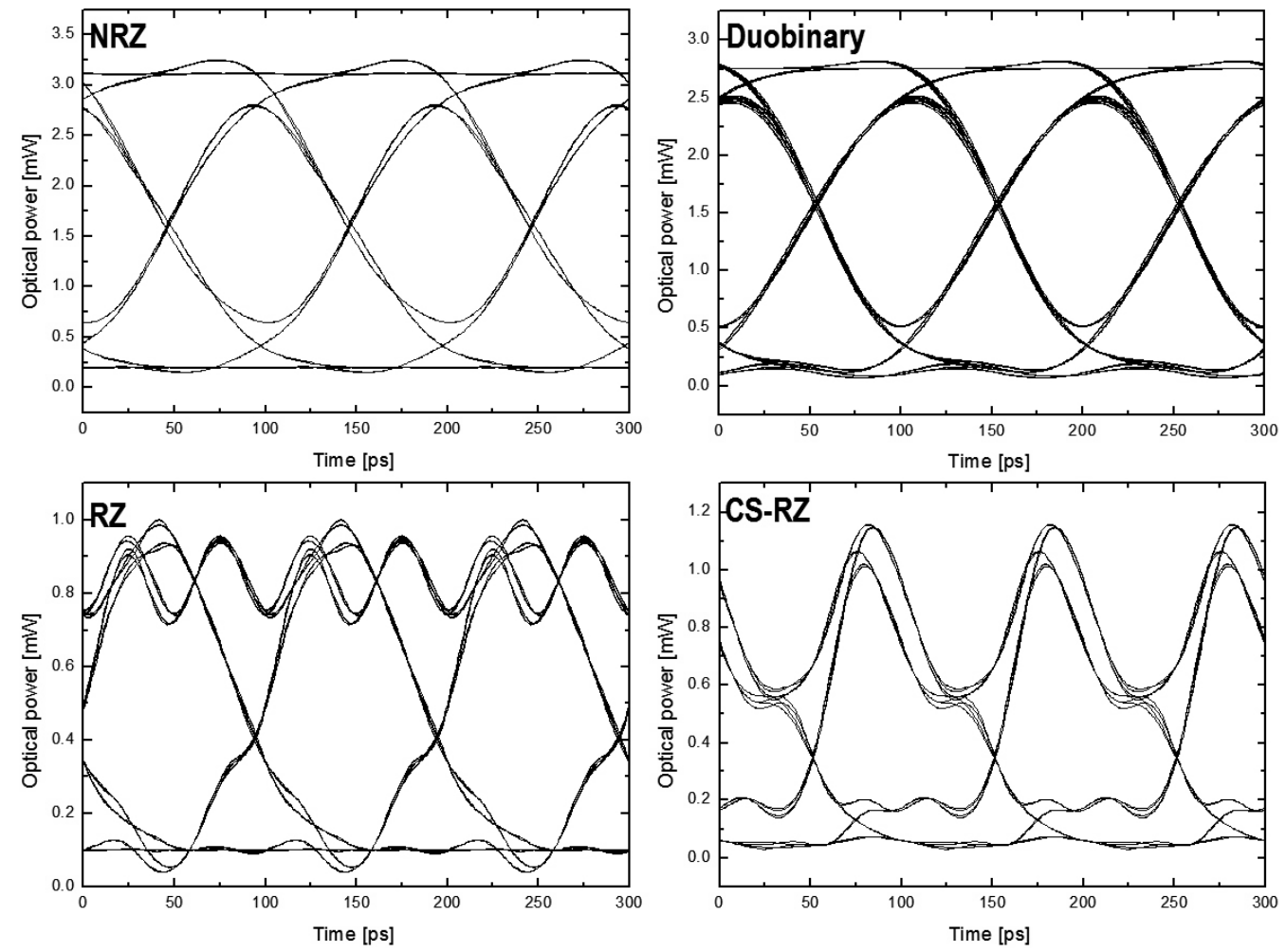

(a)
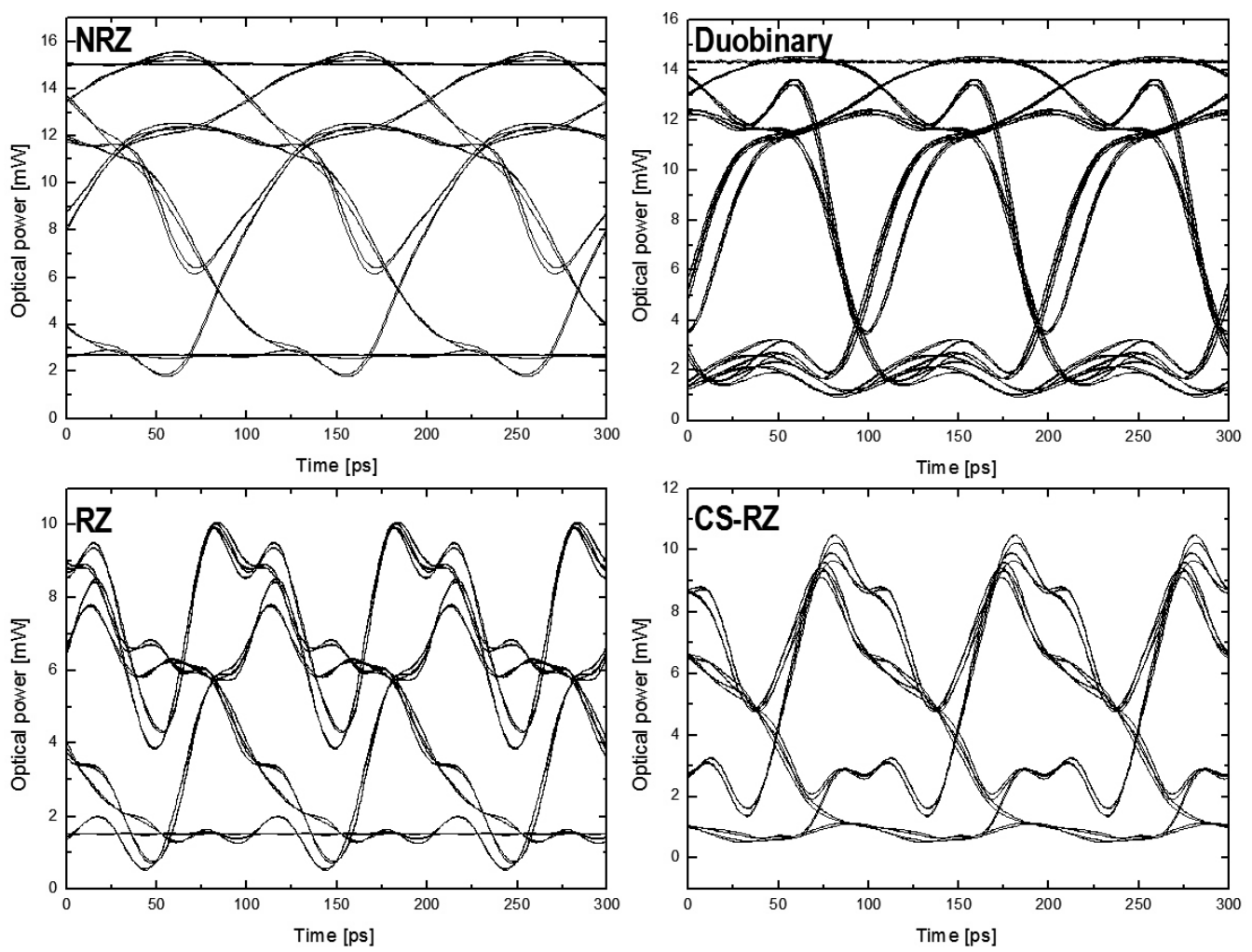

(b)

FIG. 8. Optical eye diagrams for various modulation formats; where, DGD $=50 \mathrm{ps}, \Delta \tau_{\omega}=0.57 \mathrm{ps} / \mathrm{GHz} 2|2 \vec{k}|$ and

$=2.07 \%$ GHz: (a) Without SOAs as booster amplifiers and (b) Peak-to-peak chirp $=0.4 \AA$ 
penalty, the first- and second-order PMD tolerance of the NRZ modulation format has the highest sensitivity to SOA-induced chirp, and that of the CS-RZ modulation format has the lowest sensitivity to SOA-induced chirp. Fig. 8 shows eye diagrams of modulation formats in the system with and without SOAs as booster amplifiers, where DGD $=50 \mathrm{ps}, \Delta \tau_{\omega}=0.57 \mathrm{ps} / \mathrm{GHz}$, and $2|2 \vec{k}|=$ $2.07^{\circ} / \mathrm{GHz}$. As previously mentioned, CS-RZ signals, where DGD $=50 \mathrm{ps}, \Delta \tau_{\omega}=0.57 \mathrm{ps} / \mathrm{GHz}$, and $2|2 \vec{k}|=2.07$ - $/ \mathrm{GHz}$ are slightly distorted up to a $0.4 \AA$ peak-to-peak chirp. However, NRZ and duobinary signals are severely distorted, as shown in the eye diagrams.

\section{CONCLUSIONS}

We investigated the effect of SOA-induced chirp on the PMD tolerance of various modulation formats; NRZ, duobinary NRZ, RZ, and CS-RZ. We showed that all modulation formats have very similar power penalties, induced only by first-order PMD, regardless of peak-topeak SOA-induced chirp, since the duty cycle of the signal isn't affected by SOA-induced chirp. In other words, the first-order PMD tolerance is largely unaffected by SOA-induced chirp. On the other hand, the firstand second-order PMD tolerance of all modulation formats, with the exception of the CS-RZ modulation format, are severely degraded by SOA-induced chirp. Under PMD conditions; where, DGD $=50 \mathrm{ps}, \Delta \tau_{\text {o }}=$ $0.57 \mathrm{ps} / \mathrm{GHz}$, and $2|2 \vec{k}|=2.07^{\circ} / \mathrm{GHz}$; For the NRZ modulation format, the PMD-induced power penalty increases by $3 \mathrm{~dB}$ as the peak-to-peak chirp increases from $0 \AA$ to $0.26 \AA$. For the duobinary NRZ modulation format, the PMD-induced power penalty increases by $3 \mathrm{~dB}$ as the peak-to-peak chirp increases from $0 \AA$ to $0.32 \AA$. For the RZ modulation format, the value of the peak-to-peak chirp which generates an additional $3 \mathrm{~dB}$ power penalty increases up to $0.52 \AA$. However, the PMD tolerance of the CS-RZ modulation format is degraded by $1.9 \mathrm{~dB}$ only, up to $0.6 \AA$. In conclusion, in terms of the additional $3 \mathrm{~dB}$ power penalty, the firstand second-order PMD tolerance of the NRZ modulation format has the highest sensitivity to SOA-induced chirp and that of CS-RZ modulation format has the lowest sensitivity to SOA-induced chirp.

\section{ACKNOWLEDGMENT}

This work was supported by the Brain Korea 21 Project in 2008 .

\section{REFERENCES}

[1] Henrik Sunnerud, Chongjin Xie, Magnus Karlsson, Robert Samuelsson, and Peter A. Andrekson, "A Comparison Between Different PMD Compensation Techniques,” J. Lightwave Tech., vol. 20, no. 3, pp. 368-378, 2002.

[2] Chongjin Xie, Lothar Möller, Herbert Haunstein, and Stefan Hunsche, "Comparison of System Tolerance to Polarization-Mode Dispersion Between Different Modulation Formats," IEEE Photon. Technol. Lett., vol. 15, no. 8, pp. 1168-1170, 2003.

[3] W. Kaier, S.Otte, T. Wuth, and W. Rosenkranz, "Experimental Verification of Reduced Sensitivity of Optical Duobinary Modulation to Higher Order PMD," in Tech. Dig., OFC'2002, Paper Tul5, 2002.

[4] S. Hur, Y. Kim, H. Jang, and Jichai Jeong, "10 Gbps WDM transmission performance limits using in-line SOAs and an optical phase conjugator based on four-wave mixing in SOAs as a mid-span spectral inversion technique," Opt. Exp., vol. 14, no. 11, pp. 4589- 4600, 2006.

[5] T, Y. Kim, J. Y. Kim, and S. K. Han, "All-optical Regenerator Using Semi-reflective Semiconductor Optical Amplifier," J. Opt. Soc. Korea, vol. 10, no. 1, pp. 11-15, 2006.

[6] Moon-Ki Hong,Sang-Kook Han, and Sang-Hoon Lee, "Linearization of DFB LD by using Cross Gain Modulation of Reflective SOA in Radio-over-Fiber Link," $J$. Opt. Soc. Korea, vol. 11, no. 4, pp. 158-161, 2007.

[7] T. Watanabe, N. Sakaida, H. Yasaka, and M. Koga, "Chirp control of an optical signal using phase modulation in a semiconductor optical amplifier," IEEE Photon. Technol. Lett., vol. 10, no. 7, pp. 1082-1084, 2000.

[8] W. Choi, S. Hur, J. Lee, Y. Kim, and J. Jeong, "Transmission performance analysis of $8 \times 10$ Gbps WDM signals using cascaded SOA's due to signal wavelength displacement," J. Lightwave Tech., vol. 20, no. 8, pp. 1350-1356, 2002.

[9] Cristian Francis, Frank Bruyere, Denis Penninckx, and Michel chbat, "PMD Second-Order Effects on Pulse Propagation in Single-Mode Optical Fibers," IEEE Photon. Technol. Lett., vol. 10, no. 12, pp. 1739-1741, 1998.

[10] H. Kogelnik, L. E. Nelson, and J. P. Gordon, "Emulation and Inversion of Polarization-Mode Dispersion," IEEE Photon. Technol. Lett., vol. 21, no. 2, pp. 482-495, 2003.

[11] L. E. Nelson, R. M. Jopson, H. Kogelnik, and G. J. Foschini, "Measurement of Depolarization and Scaling Associated with Second-Order Polarization Mode Dispersion in Optical Fibers," IEEE Photon. Technol. Lett., vol. 11, no. 12, pp. 1614-1616, 1999.

[12] Y. K. Park, and S. W. Granlund, "Optical Preamplifier Receivers: Application to Long-Haul Digital Transmission,” Optical Fiber Technol., vol. 1, no. 1, pp. 59-71, 1994.

[13] Rodolfo A. A. Lima, Maria Cristina R. Carvalho, and Luiz Fernando M. Conrado, "On the simulation of digital optical links with EDFA's: an accurate method for estimating BER through Gaussian approximation," IEEE J. Select. Topics Quantum Elec., vol. 3, no. 4, pp. 1037-1044, 1997. 